\title{
Ultrasound-assisted and Efficient Knoevenagel Condensation Reaction Catalyzed by Silica Sodium Carbonate Nanoparticles
}

\author{
Yaghoub Pourshojaei, ${ }^{1,2, *}$ Maryam Nikzad, ${ }^{1}$ Khalil Eskandari, ${ }^{1, *}$ Mohammad-Hossein Darijani, ${ }^{1}$ \\ Abdolreza Hassanzadeh, ${ }^{1}$ Ehsan Faghih-Mirzaei, ${ }^{1}$ Ali Asadipour ${ }^{1}$
}

\footnotetext{
1 Department of Medicinal Chemistry, Faculty of Pharmacy \& Pharmaceutics Research Center, Kerman University of Medical Sciences, Kerman, Iran

2 Neuroscience Research Center, Institute of Neuropharmacology, Kerman University of Medical Sciences, Kerman, Iran

* Corresponding author's e-mail address: y.pourshoja@kmu.ac.ir; khalileskandari@yahoo.com
}

RECEIVED: December 4, 2017 औREVISED: January 30, 2018 \ ACCEPTED: February 1, 2018

\begin{abstract}
An efficient and ultrasound-assisted route to the synthesis of arylidene malononitriles/methylciano- or ethylciano acetates in a onepot reaction catalyzed by silica sodium carbonate nanoparticles (SSC NPs) is described. In this reaction, SSC NPs demonstrated high efficiency as catalyst to obtain target products. By this achievement, a wide range of $\alpha$, $\beta$-unsaturated compounds as Knoevenagel condensation products with good to excellent yields are obtained from reaction between numerous arylaldehydes, and malononitrile, methyl cianoacetate or ethyl cianoacetate. Target products which prepared in high yield and high purity can be candidate as important biologically active molecules. This method is an easy, cheap, rapid and highly efficient for the synthesis of desired products. In addition, capability of catalyst to separate from reaction mixture and reuse in further runs and being compatible with green chemistry are considered as other advantages of this procedure. All products were deduced from their FT-IR and FT-NMR spectroscopic and elemental analysis data.
\end{abstract}

Keywords: nanocatalyst, silica sodium carbonate nanoparticles, Knoevenagel reaction, active methylene compounds.

\section{INTRODUCTION}

0 LEFINS, also called alkenes, are very important class of organic compounds that numerous natural products, herbal medicines and significant pharmacological active compounds such as vital, essential and necessary drugs may be classified into their category. ${ }^{[1-3]}$ Furthermore, olefins can be found in chemical structure of $\alpha, \beta$-unsaturated compounds. Up to date, numerous medicines and natural products have been recognized that belong to $\alpha, \beta$-unsaturated compounds. ${ }^{[4,5]}$ One of the organic reactions which lead to obtain $\alpha, \beta$-unsaturated compounds is Knoevenagel condensation reaction. ${ }^{[6-8]}$ So far this reaction extensively has been applied to the synthesis of a wide range of organic and biological active compounds. ${ }^{[9,10]}$ Also, this reaction has sometimes been used to evaluate strength of different solid basic catalysts considering the fact that $p K a$ value of active methylene compounds are about to 9-11.[11]
Knoevenagel condensations have attracted much interest by chemists and pharmacologists. Its reason may be related to importance of this reaction in organic synthesis and chemical industries. Over the past few decades, several methods have been proposed and developed by chemists for Knoevenagel condensation reactions. For instance, running the reaction in the presence of Lewis acids or bases, ${ }^{[12,13]}$ ionic liquids, ${ }^{[14]}$ green solvents, ${ }^{[15]}$ microwave assistance, ${ }^{[16]}$ ultrasound irradiation, ${ }^{[17]}$ solid-phase reactions, ${ }^{[18]}$ grinding methods, ${ }^{[19]}$ solvent-free microwave assisted conditions, ${ }^{[20]}$ and using biocatalysts, ${ }^{[15,21]}$ organocatalysts ${ }^{[22]}$ or polyoxometalates[23] could be countered as these methodologies. Furthermore, there are a few reports that have been applied to Knoevenagel condensation under different conditions including continuous flow synthesis, ${ }^{[24]}$ or fluorous biphasic system. ${ }^{[25]}$ Nevertheless, the attempts to find and propose new and efficient protocols to

(c) Br $\mathbf{B Y}$ This work is licensed under a Creative Commons Attribution 4.0 International License. 
Knoevenagel condensation reaction have been continued by chemists because all above mentioned techniques tolerate some drawbacks such as use of expensive and nonrecyclable catalysts, harsh thermal conditions, use of toxic solvents and catalysts, non-compatible with green chemistry aspects, not economically viable, and long reaction times. ${ }^{[7,8,26]}$

So far various attempts and evaluations on biological and pharmaceutical activities of $\alpha, \beta$-unsaturated compounds formed from Knoevenagel condensation reactions have been performed. For instance there are several reports that demonstrate these compounds could be consid- significant interest because of their unique advantages including easy separation from the reaction mixture and reusability, ${ }^{[37-39]}$ easy handling and storing, avoiding the generation of residual salts, nontoxicity, low solubility, increasing selectivity of the reactions, and high stability. ${ }^{[40-42]}$

Very recently a green and recyclable inexpensive Brønsted base prepared and called silica sodium carbonate (SSC) $3,{ }^{[43]}$ via a cascade two steps method from silica gel (1) and silica chloride 2 (Scheme 1). SSC has several important advantages including environmentally benign, reusability, easy handling, high perdurability, and convenience work-up.

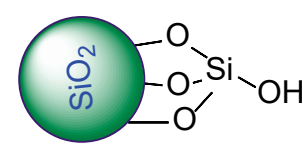

1

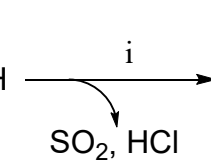

$\mathrm{SO}_{2}, \mathrm{HCl}$

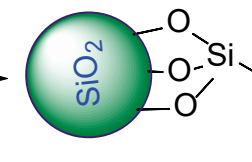

2

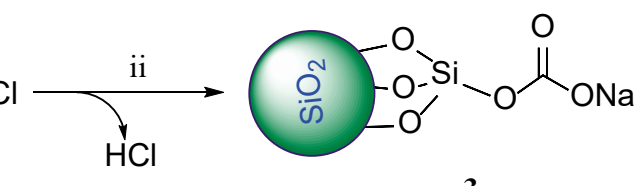

3

i) $\mathrm{SOCl}_{2}$, Reflux, $48 \mathrm{~h}$; ii) $\mathrm{NaHCO}_{3}, \mathrm{n}$-Hexane, Reflux, $24 \mathrm{~h}$

Scheme 1. Two steps route to preparation of SSC.

ered as anti-nociceptive agents modulating the TREK-1 channel, ${ }^{[27,28]}$ inhibitors of 12-Lipoxygenase, ${ }^{[29]}$ new group of fungicidal and acaricidal agents, ${ }^{[30]}$ a new class of antagonists at the glycine site of the NMDA receptor, ${ }^{[31]}$ an ingredient in sunscreens and cosmetics, ${ }^{[32]}$ herbicidally active compounds, ${ }^{[33]}$ and also a therapeutic agent for diseases of the circulatory system. ${ }^{[34]}$ Also the importance of Knoevenagel condensation products can be more realized by considering to the fact that some of these molecules have been recognized as molecular rotors ${ }^{[35]}$ which were subjected to 2016's noble prize awarded to Sauvage, Stoddartand, Feringa for their design and production of
Further to our last studies on the designing new protocols to high efficient catalytic green synthesis of novel organic compounds, ${ }^{[44]}$ and investigation of their biological activities $^{[45]}$ herein we wish to report a highly efficient and practical approach to Knoevenagel condensation reaction between a wide range of arylaldehydes 1 and malononitrile or methyl(ethyl)cianoacetate $\mathbf{2}$ using SSC NPs as an ecofriendly heterogeneous recyclable catalyst (Scheme 2). In addition, it is notable to mention that in this work by a slight modification on previous reported method, ${ }^{[43]}$ we prepared SSC nanoparticles instead of bulk one and applied it successfully in Knoevenagel condensation reaction (Scheme 2).<smiles>O=Cc1ccc(Cl)cc1</smiles>

1

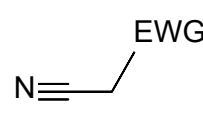

2<smiles>CC(C)(C)/C=C/c1ccc(Cl)cc1</smiles>

EWG: CN, $\mathrm{COOCH}_{3}, \mathrm{COOC}_{2} \mathrm{H}_{5}$

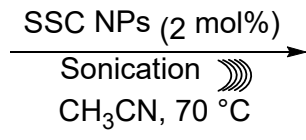

Scheme 2. Knoevenagel condensation reaction catalyzed by SSC NPS.

molecular machines. These disclosed properties encourage chemists and pharmacologists to design and synthesize of newly prepared molecules exhibiting pharmacological and biological activities which may be applied in treatment of human diseases as new medicines in the future. ${ }^{[9,10,36]}$

Recently, solid supported catalysts have attracted

\section{EXPERIMENTAL SECTION}

All chemicals were purchased from Sigma-Aldrich and Merck chemical companies. Melting points were measured on an electrothermal IA9100 melting point apparatus fixed at $1{ }^{\circ} \mathrm{C} / \mathrm{min}$. IR spectra were recorded by Brucker FT-IR 
Tensor 27 infrared spectrophotometer with $\mathrm{KBr}$ as matrix. A cylindrical planetary ball mill apparatus with a $500 \mathrm{~mL}$ jar volume made of welded steel supplied with three $19 \mathrm{~mm}$ diameter hardened steel balls (DECO-SIO-0.5L, Hunan, Mainland) was used for grinding silica gel. ${ }^{1} \mathrm{H}$ NMR and ${ }^{13} \mathrm{C}$ NMR spectra were recorded on a FT-NMR Bruker Avance Ultra Shield Spectrometer at frequencies of 400.13 and 100.62 $\mathrm{MHz}$ respectively in $\mathrm{CHCl}_{3}$ as solvent. Elemental analyses (C, H, N, S) were performed on a Heraeus Rapid analyzer and the results were found in good agreement $( \pm 0.3 \%)$ with the calculated values. Scanning electron microscopy (SEM) evaluations of the nano-catalyst were performed on a JEOL JEM 3010 instrument operating at an accelerating voltage of $300 \mathrm{kV}$. Transmission electron microscopy (TEM) investigations of the nano-catalyst were performed on a JEOL JEM 3010 instrument operating at an accelerating voltage of $300 \mathrm{kV}$. TLC was performed on TLCGrade silica gel-G/UV $254 \mathrm{~nm}$ plates EtOAc/n-hexane (1:1) as eluent. All products were isolated, purified and deduced from their IR, ${ }^{1} \mathrm{H} N M R$, and ${ }^{13} \mathrm{C}$ NMR spectral data along with elemental analyses $(\mathrm{C}, \mathrm{H}, \mathrm{N})$. The products were isolated and characterized by physical and spectral data and for known compounds they were compared with authentic samples (Table 2).

\section{Typical Procedure for the Preparation of SSC NPS}

Silica gel $60(63-200 \mu \mathrm{m})(15 \mathrm{~g})$ was grinded by a planetary ball mill apparatus under dry milling conditions for $6 \mathrm{~h}$ without using any milling additives to obtain a much finer powder. Afterwards resulted finer $\mathrm{SiO}_{2}$ was dried at $120^{\circ} \mathrm{C}$ for $6 \mathrm{~h}$. In the next step, dried finer $\mathrm{SiO}_{2}(7 \mathrm{~g})$ was added to a $100 \mathrm{~mL}$ two necked round bottom flask put previously into an ice bath. Then, thionyl chloride $(28 \mathrm{~mL}$ ) was added dropwise to the flask (caution). One of the flask's necks was connected to a pot of water by a hose for smoking out of produced $\mathrm{HCl}(\mathrm{g})$. When the addition of thionyl chloride was completed, the other flask's neck was connected to a reflux condenser and it was allowed to the reaction mixture to stir for $0.5 \mathrm{~h}$ at room temperature and then for $48 \mathrm{~h}$ under reflux conditions respectively. Afterwards, the reaction mixture was cooled to room temperature and filtered to obtain silica chloride. In second step, obtained silica chloride ( $5 \mathrm{~g}$ ) which was previously dried at $120^{\circ} \mathrm{C}$ for $6 \mathrm{~h}$ was added to a stirred $100 \mathrm{~mL}$ round bottom flask containing sodium bicarbonate $(5 \mathrm{~g})$ and dry $n$-hexane $(20 \mathrm{~mL})$ under reflux conditions. After enough time passing $(24 \mathrm{~h})$, the reaction mixture was filtered to separate the catalyst. Then to remove the remaining sodium bicarbonate, the catalyst was washed with $5 \mathrm{~mL}$ of distilled water for ten times $(10 \times 5$ $\mathrm{mL}$ ) so the filtrate was neutral. Finally the catalyst was dried at $90^{\circ} \mathrm{C}$ for $6 \mathrm{~h}$ and then was grinded again by a ball mill for $1 \mathrm{~h}$ to obtain SSC NPs.

\section{General Procedure for the Preparation of Corresponding Aldehydes 1}

Corresponding aldehydes containing alkylamine ethers was synthesized according to the procedure reported in the literature, ${ }^{[45 a]}$ and all structures were confirmed by comparison with their physical and chemical data reported in literature. Also $p$-benziloxybenzaldehyde derivatives were prepared by treated with 4-hydroxybenzaldehyde with appropriate benzylchloride/bromide derivatives in the presence of $\mathrm{K}_{2} \mathrm{CO}_{3}$ as catalyst. The physical and chemical properties of all obtained substrates were compared with the ones reported in literature and all of their structures were confirmed. ${ }^{[46]}$

\section{Typical Procedure to the Synthesis of 3c}

4-morpholinobenzaldehyde ( $1 \mathrm{mmol}, 0.191 \mathrm{~g}$ ) was added to a stirred mixture of malononitrile $(1.5 \mathrm{mmol}, 0.099 \mathrm{~g})$, and catalytic amount of SSC NPs (0.012 g, $2 \mathrm{~mol} \%$ ) in acetonitrile $(10 \mathrm{~mL})$. It was allowed to the mixture to stir at 70 ${ }^{\circ} \mathrm{C}$ under sonication about 25-60 minutes. After completion of the reaction (the reaction progress was monitored by TLC using EtOAc/ $n$-hexane (1:1) as eluent), the reaction mixture was filtered to separate precipitate. Next, the precipitate was dissolved in boiling ethanol and then was filtrated to separate catalyst. Finally, pure crystalline product was obtained from filtrate. Since the catalyst is reusable, at the end of the reaction, it was washed by boiling methanol three times $(3 \times 2 \mathrm{~mL})$, dried at $90^{\circ} \mathrm{C}$ for $2 \mathrm{~h}$ and re-used in further cycles. Also, in the following, we explain more details about reusability results of the catalyst on model reaction.

\section{Representative Spectral Data}

2-(4-Morpholinobenzylidene)malononitrile (3c): m.p. 198$200^{\circ} \mathrm{C}$; IR (KBr) $\left(v_{\max } / \mathrm{cm}^{-1}\right): 3008,2955,2921,2889,2217$, 1606, 1568, 1518, 1446, 1397, 1238, 1196, 928, 824, 621. ${ }^{1} \mathrm{H}$ NMR $\left(300 \mathrm{MHz}, \mathrm{CDCl}_{3}\right) \delta / \mathrm{ppm}: 7.85(2 \mathrm{H}, \mathrm{d}, J=9 \mathrm{~Hz}$, $\left.\mathrm{CH}_{\mathrm{Ar}}\right), 7.51\left(1 \mathrm{H}, \mathrm{s}, \mathrm{CH}_{\text {vinyl }}\right), 6.89\left(2 \mathrm{H}, \mathrm{d}, J=9 \mathrm{~Hz}, \mathrm{CH}_{\mathrm{Ar}}\right), 3.88$ $\left(4 \mathrm{H}, \mathrm{t}, J=6 \mathrm{~Hz}, \mathrm{CH}_{2} \mathrm{OCH}_{2}\right), 3.45\left(4 \mathrm{H}, \mathrm{t}, J=6 \mathrm{~Hz}, \mathrm{CH}_{2} \mathrm{NCH}_{2}\right)$. ${ }^{13} \mathrm{CNMR}\left(75 \mathrm{MHz}, \mathrm{CDCl}_{3}\right) \delta / \mathrm{ppm}: 158.2,154.7,133.7$, 121.2, 115.5, 114.4, 113.2, 74.4, 66.3, 46.6. Anal. Calcd. $w / \%$ for $\mathrm{C}_{14} \mathrm{H}_{13} \mathrm{~N}_{3} \mathrm{O}$ (239.28): C, 70.28; $\mathrm{H}, 5.48 ; \mathrm{N}, 17.56$; Found: $\mathrm{C}, 70.35 ; \mathrm{H}, 5.41 ; \mathrm{N}, 17.44$.

2-(4-(Piperidin-1-yl)benzylidene)malononitrile (3d): m.p. 134-135 ${ }^{\circ} \mathrm{C}$; IR (KBr) $\left(v_{\max } / \mathrm{cm}^{-1}\right): 2939,2858,2824,2215$, $1607,1566,1515,1441,1362,1246,1198,1127,932,853$, 606. ${ }^{1} \mathrm{H}$ NMR (300 MHz, $\left.\mathrm{CDCl}_{3}\right) \delta / \mathrm{ppm}: 8.04\left(1 \mathrm{H}, \mathrm{s}, \mathrm{CH}_{\text {vinylic }}\right)$, $7.82\left(2 \mathrm{H}, \mathrm{d}, J=9 \mathrm{~Hz}, \mathrm{CH}_{\mathrm{Ar}}\right), 7.05\left(2 \mathrm{H}, \mathrm{d}, J=9 \mathrm{~Hz}, \mathrm{CH}_{\mathrm{Ar}}\right), 3.54$ $\left(4 \mathrm{H}\right.$, brs, $\left.\mathrm{CH}_{2} \mathrm{NCH}_{2}\right), 1.58\left(6 \mathrm{H}\right.$, brs, $\left.3 \times \mathrm{CH}_{2}\right) .{ }^{13} \mathrm{CNMR}(75$ $\left.\mathrm{MHz}, \mathrm{CDCl}_{3}\right) \delta / \mathrm{ppm}: 159.1,154.6,134.3,119.6,116.7$, 115.9, 113.4, 69.6, 47.8, 25.6, 24.4. Anal. Calcd. $w / \%$ for $\mathrm{C}_{15} \mathrm{H}_{15} \mathrm{~N}_{3}$ (237.31): C, 75.92; $\mathrm{H}, 6.37 ; \mathrm{N}, 17.71$; Found: $\mathrm{C}$, 75.88; H, 6.35; N, 17.80 . 
2-(4-(4-Phenylpiperidin-1-yl)benzylidene)malononitrile (3e): m.p. $127-128^{\circ} \mathrm{C}$; IR $(\mathrm{KBr})\left(v_{\max } / \mathrm{cm}^{-1}\right)$ : 3008, 2930, 2883, 2215, 1605, 1561, 1509, 1447, 1406, 1355, 1196, 1073, 1053, 964, 820, 606. ${ }^{1} \mathrm{HNMR}\left(300 \mathrm{MHz}\right.$, DMSO- $d_{6}$ ) $\delta / \mathrm{ppm}$ : $8.05\left(1 \mathrm{H}, \mathrm{s}, \mathrm{CH}_{\text {vinylic }}\right), 7.82\left(2 \mathrm{H}, \mathrm{d}, J=9 \mathrm{~Hz}, \mathrm{CH}_{\mathrm{Ar}}\right), 7.32-7.17$ $\left(5 \mathrm{H}, \mathrm{m}, \mathrm{CH}_{\mathrm{Ph}}\right), 7.05\left(2 \mathrm{H}, \mathrm{d}, J=9 \mathrm{~Hz}, \mathrm{CH}_{\mathrm{Ar}}\right), 4.10(2 \mathrm{H}, \mathrm{d}, J=12$ $\left.\mathrm{Hz}, \mathrm{CH}_{2}\right), 2.95\left(2 \mathrm{H}, \mathrm{t}, J=12 \mathrm{~Hz}, \mathrm{CH}_{2}\right), 1.89-1.82(1 \mathrm{H}, \mathrm{m}$, $\left.\mathrm{CH}_{\mathrm{Bn}}\right), 1.65\left(2 \mathrm{H}, \mathrm{d}, J=12 \mathrm{~Hz}, \mathrm{CH}_{2}\right), 1.24-1.11\left(2 \mathrm{H}, \mathrm{m}, \mathrm{CH}_{2}\right)$. ${ }^{13} \mathrm{CNMR}\left(75 \mathrm{MHz}\right.$, DMSO- $d_{6}$ ) $\delta /$ ppm: 159.1, 154.5, 140.4, $134.3,129.4,128.6,126.3,119.7,116.7,115.8,113.5,69.8$, 47.0, 42.4, 37.8, 31.6. Anal. Calcd. $w / \%$ for $\mathrm{C}_{21} \mathrm{H}_{19} \mathrm{~N}_{3}$ (313.40) C, 80.48; H, 6.11; N, 13.41; Found: C, 80.57; H, 6.09; N, 13.49.

2-(4-(2-Morpholinoethoxy)benzylidene)malononitrile (3f): m.p. $228-229^{\circ} \mathrm{C}$; IR (KBr) $\left(v_{\max } / \mathrm{cm}^{-1}\right)$ : 3005, 2970, 2938, 2571, 2538, 2449, 2221, 1603, 1587, 1557, 1511, 1430, $1268,1182,1080,943,831,612 .{ }^{1 H N M R}$ (300 MHz, DMSO$\left.d_{6}\right) \delta / \mathrm{ppm}: 8.45\left(1 \mathrm{H}, \mathrm{s}, \mathrm{CH}_{\text {vinylic }}\right), 8.01\left(2 \mathrm{H}, \mathrm{d}, J=9 \mathrm{~Hz}, \mathrm{CH}_{\mathrm{Ar}}\right)$, $7.25\left(2 \mathrm{H}, \mathrm{d}, J=9 \mathrm{~Hz}, \mathrm{CH}_{\mathrm{Ar}}\right), 4.53\left(2 \mathrm{H}, \mathrm{t}, J=6 \mathrm{~Hz}, \mathrm{OCH}_{2}\right), 3.84$ $\left(4 \mathrm{H}, \mathrm{t}, J=6 \mathrm{~Hz}, \mathrm{CH}_{2} \mathrm{OCH}_{2}\right), 3.40\left(2 \mathrm{H}, \mathrm{brs}, \mathrm{CH}_{2} \mathrm{~N}\right), 3.15(4 \mathrm{H}$, brs, $\mathrm{CH}_{2} \mathrm{NCH}_{2}$ ). ${ }^{13} \mathrm{CNMR}\left(75 \mathrm{MHz}\right.$, DMSO- $\left.d_{6}\right) \delta /$ ppm: 163.1, 160.9, 133.7, 125.1, 116.3, 115.2, 114.3, 77.8, 64.3, 64.0, 55.5, 52.5. Anal. Calcd. $w / \%$ for $\mathrm{C}_{16} \mathrm{H}_{17} \mathrm{~N}_{3} \mathrm{O}_{2}$ (283.33) C, $67.83 ; \mathrm{H}, 6.05 ; \mathrm{N}, 14.83$; Found: C, 67.87; H, 6.00; N, 14.89 .

2-(3-(2-Morpholinoethoxy)benzylidene)malononitrile (3g): m.p. $225-226{ }^{\circ} \mathrm{C}$; IR (KBr) $\left(v_{\max } / \mathrm{cm}^{-1}\right)$ : 3005, 2972, 2925, 2663, 2455, 2228, 1572, 1450, 1270, 1185, 1128, 922, 860, 684. ${ }^{1} \mathrm{HNMR}\left(300 \mathrm{MHz}, \mathrm{DMSO}-d_{6}\right) \delta / \mathrm{ppm}: 8.56(1 \mathrm{H}, \mathrm{s}$, $\left.\mathrm{CH}_{\text {vinylic }}\right), 7.61-7.56\left(3 \mathrm{H}, \mathrm{m}, \mathrm{CH}_{\mathrm{Ar}}\right), 7.39-7.36\left(1 \mathrm{H}, \mathrm{m}, \mathrm{CH}_{\mathrm{Ar}}\right)$, $4.51\left(2 \mathrm{H}, \mathrm{t}, J=6 \mathrm{~Hz}, \mathrm{OCH}_{2}\right), 3.90\left(4 \mathrm{H}, \mathrm{brs}, \mathrm{CH}_{2} \mathrm{OCH}_{2}\right), 3.52$ $\left(2 \mathrm{H}\right.$, brs, $\left.\mathrm{CH}_{2} \mathrm{~N}\right), 3.28\left(4 \mathrm{H}\right.$, brs, $\left.\mathrm{CH}_{2} \mathrm{NCH}_{2}\right) .{ }^{13} \mathrm{CNMR}(75 \mathrm{MHz}$, DMSO- $\left.d_{6}\right) \delta /$ ppm: 161.7, 158.3, 133.0, 131.3, 123.8, 121.2, 116.7, 114.6, 113.6, 82.6, 63.8, 63.4, 55.2, 52.2. Anal. Calcd. $w / \%$ for $\mathrm{C}_{16} \mathrm{H}_{17} \mathrm{~N}_{3} \mathrm{O}_{2}$ (283.33) C, 67.83; $\mathrm{H}, 6.05 ; \mathrm{N}, 14.83$; Found: C, 67.89; H, 5.97; N, 14.85 .

2-(4-(2-(Piperidin-1-yl)ethoxy)benzylidene)malononitrile (3h): m.p. $195-198{ }^{\circ} \mathrm{C}$; IR (KBr) $\left(v_{\max } / \mathrm{cm}^{-1}\right): 3002,2944,2859$, 2612, 2449, 2388, 2226, 1603, 1585, 1559, 1459, 1260, 1190, 1018, 837, 613. ${ }^{1} \mathrm{H}$ NMR (300 MHz, DMSO- $\left.d_{6}\right) \delta /$ ppm: $8.45\left(1 \mathrm{H}, \mathrm{s}, \mathrm{CH}_{\text {vinylic }}\right), 7.99\left(2 \mathrm{H}, \mathrm{d}, J=9 \mathrm{~Hz}, \mathrm{CH}_{\mathrm{Ar}}\right), 7.23$ $\left(2 \mathrm{H}, \mathrm{d}, J=9 \mathrm{~Hz}, \mathrm{CH}_{\mathrm{Ar}}\right), 4.50\left(2 \mathrm{H}, \mathrm{t}, J=6 \mathrm{~Hz}, \mathrm{OCH}_{2}\right), 3.26(2 \mathrm{H}$, brs, $\left.\mathrm{CH}_{2} \mathrm{~N}\right), 3.01\left(4 \mathrm{H}\right.$, brs, $\left.\mathrm{CH}_{2} \mathrm{NCH}_{2}\right), 1.73\left(4 \mathrm{H}\right.$, brs, $\left.2 \mathrm{CH}_{2}\right)$, $1.49\left(2 \mathrm{H}\right.$, brs, $\mathrm{CH}_{2}$ ). ${ }^{13} \mathrm{CNMR}$ (75 MHz, DMSO- $\left.d_{6}\right) \delta / \mathrm{ppm}$ : 163.3, 160.9, 133.8, 124.9, 116.2, 115.2, 114.3, 77.7, 64.4, 55.5, 53.4, 23.6, 22.4. Anal. Calcd. $w / \%$ for $\mathrm{C}_{17} \mathrm{H}_{19} \mathrm{~N}_{3} \mathrm{O}$ (281.36) C, 72.57; H, 6.81; N, 14.94; Found: C, 72.65; H, $6.75 ; \mathrm{N}, 14.97$.

2-(4-((4-Chlorobenzyl)oxy)benzylidene)malononitrile (3i): m.p. $174-176^{\circ} \mathrm{C}$; IR (KBr) $\left(v_{\max } / \mathrm{cm}^{-1}\right)$ : 3032, 2924, 2853, 2221, 1603, 1582, 1556, 1509, 1433, 1274, 1237, 1178,
$1013,828,798,594 .{ }^{1} \mathrm{HNMR}\left(300 \mathrm{MHz}, \mathrm{DMSO}-d_{6}\right) \delta / \mathrm{ppm}$ : $8.41\left(1 \mathrm{H}, \mathrm{s}, \mathrm{CH}_{\text {vinylic }}\right), 7.99\left(2 \mathrm{H}, \mathrm{d}, J=9 \mathrm{~Hz}, \mathrm{CH}_{\mathrm{Ar}}\right), 7.53-7.46$ $\left(4 \mathrm{H}, \mathrm{m}, \mathrm{CH}_{\mathrm{Ar}}\right), 7.27\left(2 \mathrm{H}, \mathrm{d}, \mathrm{J}=9 \mathrm{~Hz}, \mathrm{CH}_{\mathrm{Ar}}\right), 5.26\left(2 \mathrm{H}, \mathrm{s}, \mathrm{OCH}_{2}\right)$. ${ }^{13} \mathrm{CNMR}\left(75 \mathrm{MHz}\right.$, DMSO-d $\left.d_{6}\right) \delta / \mathrm{ppm}: 163.6,160.8,135.5$, $133.8,133.2,130.2,129.0,124.8,116.4,115.7,115.2$, 114.3, 77.6, 69.4. Anal. Calcd. $w / \%$ for $\mathrm{C}_{17} \mathrm{H}_{11} \mathrm{ClN}_{2} \mathrm{O}$ (294.74): C, 69.28; H, 3.76; N, 9.50; Found: C, 69.25; H, 3.77; N, 9.55 .

2-(3-((4-Chlorobenzyl)oxy)benzylidene)malononitrile (3j): m.p. $100-103^{\circ} \mathrm{C}$; IR (KBr) $\left(v_{\max } / \mathrm{cm}^{-1}\right): 3040,2926,2224$, 1602, 1585, 1493, 1451, 1378, 1270, 1185, 1015, 801, 675. ${ }^{1} \mathrm{H}$ NMR (300 MHz, $\left.\mathrm{CDCl}_{3}\right) \delta / \mathrm{ppm}: 7.76\left(1 \mathrm{H}, \mathrm{s}, \mathrm{CH}_{\text {vinylic }}\right), 7.59$ $\left(1 \mathrm{H}, \mathrm{s}, \mathrm{CH}_{\mathrm{Ar}}\right), 7.52-7.41\left(6 \mathrm{H}, \mathrm{m}, \mathrm{CH}_{\mathrm{Ar}}\right), 7.30-7.24(1 \mathrm{H}, \mathrm{m}$, $\left.\mathrm{CH}_{\mathrm{Ar}}\right), 5.13\left(2 \mathrm{H}, \mathrm{s}, \mathrm{OCH}_{2}\right) .{ }^{13} \mathrm{CNMR}\left(75 \mathrm{MHz}, \mathrm{CDCl}_{3}\right) \delta / \mathrm{ppm}$ : $159.7,159.0,134.4,134.2,132.1,130.7,128.9,124.5$, 122.2, 114.8, 113.6, 112.6, 83.1, 69.5. Anal. Calcd. $w / \%$ for $\mathrm{C}_{17} \mathrm{H}_{11} \mathrm{ClN}_{2} \mathrm{O}$ (294.74): C, 69.28; H, 3.76; N, 9.50; Found: C, 69.33; H, 3.71; N, 9.61.

2-(4-((4-Bromobenzyl)oxy)benzylidene)malononitrile (3k): m.p. $187-190^{\circ} \mathrm{C}$; IR (KBr) $\left(v_{\max } / \mathrm{cm}^{-1}\right)$ : 3029, 2220, 1604, $1582,1555,1507,1432,1272,1236,1176,1070,1010,827$, 609. ${ }^{1} \mathrm{HNMR}\left(300 \mathrm{MHz}, \mathrm{CDCl}_{3}\right) \delta / \mathrm{ppm}: 8.41\left(1 \mathrm{H}, \mathrm{s}, \mathrm{CH}_{\text {vinylic }}\right)$, $7.99\left(2 \mathrm{H}, \mathrm{d}, J=9 \mathrm{~Hz}, \mathrm{CH}_{\mathrm{Ar}}\right), 7.62\left(2 \mathrm{H}, \mathrm{d}, J=9 \mathrm{~Hz}, \mathrm{CH}_{\mathrm{Ar}}\right), 7.44$ $\left(2 \mathrm{H}, \mathrm{d}, J=9 \mathrm{~Hz}, \mathrm{CH}_{\mathrm{Ar}}\right), 7.27\left(2 \mathrm{H}, \mathrm{d}, J=9 \mathrm{~Hz}, \mathrm{CH}_{\mathrm{Ar}}\right), 5.25(2 \mathrm{H}$, s, $\left.\mathrm{OCH}_{2}\right) .{ }^{13} \mathrm{CNMR}\left(75 \mathrm{MHz}, \mathrm{CDCl}_{3}\right) \delta / \mathrm{ppm}: 163.6,160.8$, 135.9, 133.8, 131.9, 130.5, 124.8, 121.8, 116.4, 115.2, 114.3, 77.6, 69.5. Anal. Calcd. $w / \%$ for $\mathrm{C}_{17} \mathrm{H}_{11} \mathrm{BrN}_{2} \mathrm{O}$ (339.19): C, $60.20 ; \mathrm{H}, 3.27$; N, 8.26; Found: C, 60.25; H, 3.19; N, 8.31.

2-(3-((4-Bromobenzyl)oxy)benzylidene)malononitrile (3I): m.p. $104-105^{\circ} \mathrm{C}$; IR (KBr) $\left(v_{\max } / \mathrm{cm}^{-1}\right)$ : 3037, 2929, 2223, $1601,1574,1487,1451,1272,1185,1070,1013,844,806$, 786, 619. ${ }^{1} \mathrm{HNMR}\left(300 \mathrm{MHz}, \mathrm{CDCl}_{3}\right) \delta / \mathrm{ppm}: 8.51(1 \mathrm{H}, \mathrm{s}$, $\left.\mathrm{CH}_{\text {vinylic }}\right), 7.62\left(1 \mathrm{H}, \mathrm{s}, \mathrm{CH}_{\mathrm{Ar}}\right), 7.60-7.55\left(4 \mathrm{H}, \mathrm{m}, \mathrm{CH}_{\mathrm{Ar}}\right), 7.43$ $\left(2 \mathrm{H}, \mathrm{d}, J=6 \mathrm{~Hz}, \mathrm{CH}_{\mathrm{Ar}}\right), 7.38-7.34\left(1 \mathrm{H}, \mathrm{m}, \mathrm{CH}_{\mathrm{Ar}}\right), 5.16(2 \mathrm{H}, \mathrm{s}$, $\mathrm{OCH}_{2}$ ). ${ }^{13} \mathrm{CNMR}$ (75 MHz, $\mathrm{CDCl}_{3}$ ) $\delta / \mathrm{ppm}: 161.7,158.8$, $136.2,132.9,131.9,131.2,130.4,123.6,121.7,121.2$, 116.9, 114.6, 113.6, 82.4, 69.2. Anal. Calcd. $w / \%$ for $\mathrm{C}_{17} \mathrm{H}_{11} \mathrm{BrN}_{2} \mathrm{O}$ (339.19): C, 60.20; H, 3.27; N, 8.26; Found: $\mathrm{C}$, $60.27 ; \mathrm{H}, 3.21 ; \mathrm{N}, 8.33$.

Methyl (E)-2-cyano-3-(4-morpholinophenyl)acrylate (3n): m.p. $146-149^{\circ} \mathrm{C}$; IR (KBr) $\left(v_{\max } / \mathrm{cm}^{-1}\right): 2953,2879,2851$, 2215, 1719, 1613, 1575, 1523, 1443, 1400, 1284, 1230, 1192, 1051, 925, 813, 614, 502. ${ }^{1} \mathrm{HNMR}\left(300 \mathrm{MHz}, \mathrm{CDCl}_{3}\right) \delta$ / ppm: $8.12\left(1 \mathrm{H}, \mathrm{s}, \mathrm{CH}_{\text {vinyli }} \mathrm{C}\right), 7.97\left(2 \mathrm{H}, \mathrm{d}, J=9 \mathrm{~Hz}, \mathrm{CH}_{\text {Ar }}\right), 6.90$ $\left(2 \mathrm{H}, \mathrm{d}, J=9 \mathrm{~Hz}, \mathrm{CH}_{\mathrm{Ar}}\right), 3.92\left(3 \mathrm{H}, \mathrm{s}, \mathrm{OCH}_{3}\right), 3.88(4 \mathrm{H}, \mathrm{t}, J=6$ $\left.\mathrm{Hz}, \mathrm{CH}_{2} \mathrm{OCH}_{2}\right), 3.40\left(4 \mathrm{H}, \mathrm{t}, J=6 \mathrm{HZ}, \mathrm{CH}_{2} \mathrm{NCH}_{2}\right) .{ }^{13} \mathrm{CNMR}(75$ $\left.\mathrm{MHz}, \mathrm{CDCl}_{3}\right) \delta$ / ppm: 164.2, 154.4, 154.1, 133.8, 121.6, 116.9, 113.5, 96.0, 66.4, 53.0, 46.9. Anal. Calcd. $w / \%$ for $\mathrm{C}_{15} \mathrm{H}_{16} \mathrm{~N}_{2} \mathrm{O}_{3}$ (272.30): C, 66.16; H, 5.92; N, 10.29; Found: $\mathrm{C}$, $66.23 ; \mathrm{H}, 5.87 ; \mathrm{N}, 10.35$. 
Methyl (E)-2-cyano-3-(4-(piperidin-1-yl)phenyl)acrylate (30): m.p. 96-99 ${ }^{\circ} \mathrm{C}$; IR $(\mathrm{KBr})\left(v_{\max } / \mathrm{cm}^{-1}\right): 3022,3002,2936$, 2851, 2215, 1714, 1612, 1573, 1523, 1416, 1361, 1281, $1226,1182,1131,1092,1015,808,759,578,496 .{ }^{1} \mathrm{H}$ NMR (300 MHz, $\left.\mathrm{CDCl}_{3}\right) \delta /$ ppm: $7.98\left(1 \mathrm{H}, \mathrm{s}, \mathrm{CH}_{\text {vinylic }}\right), 7.83(2 \mathrm{H}, \mathrm{d}$, $\left.J=9 \mathrm{~Hz}, \mathrm{CH}_{\mathrm{Ar}}\right), 6.77\left(2 \mathrm{H}, \mathrm{d}, J=9 \mathrm{~Hz}, \mathrm{CH}_{\mathrm{Ar}}\right), 3.80\left(3 \mathrm{H}, \mathrm{s}, \mathrm{OCH}_{3}\right)$, $3.37\left(4 \mathrm{H}\right.$, brs, $\left.\mathrm{CH}_{2} \mathrm{NCH}_{2}\right), 1.60\left(6 \mathrm{H}, \mathrm{brs}, 3 \times \mathrm{CH}_{2}\right) .{ }^{13} \mathrm{CNMR}(75$ $\left.\mathrm{MHz}, \mathrm{CDCl}_{3}\right) \delta$ / ppm: 164.7, 154.4, 154.0, 134.2, 120.0, 117.4, 113.2, 94.0, 52.8, 48.1, 25.3, 24.3. Anal. Calcd. $w / \%$ for $\mathrm{C}_{16} \mathrm{H}_{18} \mathrm{~N}_{2} \mathrm{O}_{2}$ (270.33): C, 71.09; $\mathrm{H}, 6.71 ; \mathrm{N}, 10.36$; Found: C, $71.15 ; \mathrm{H}, 6.68 ; \mathrm{N}, 10.33$.

Methyl (E)-2-cyano-3-(4-(2-morpholinoethoxy)phenyl)acrylate (3p): m.p. 203-207 ${ }^{\circ} \mathrm{C}$; IR (KBr) $\left(v_{\max } / \mathrm{cm}^{-1}\right)$ : 2957, 2925, 2224, 1726, 1599, 1512, 1434, 1274, 1212, 1185, 1127, 943, 838, 526. ${ }^{1} \mathrm{HNMR}\left(300 \mathrm{MHz}, \mathrm{DMSO}-d_{6}\right) \delta / \mathrm{ppm}: 8.33(1 \mathrm{H}$, $\left.\mathrm{s}, \mathrm{CH}_{\text {vinylic }}\right), 8.09\left(2 \mathrm{H}, \mathrm{d}, J=9 \mathrm{~Hz}, \mathrm{CH}_{\mathrm{Ar}}\right), 7.20(2 \mathrm{H}, \mathrm{d}, J=9 \mathrm{~Hz}$, $\left.\mathrm{CH}_{\mathrm{Ar}}\right), 4.32\left(2 \mathrm{H}, \mathrm{t}, J=6 \mathrm{~Hz}, \mathrm{OCH}_{2}\right), 3.86\left(3 \mathrm{H}, \mathrm{s}, \mathrm{OCH}_{3}\right), 3.67$ $\left(4 \mathrm{H}, \mathrm{t}, J=6 \mathrm{~Hz}, \mathrm{CH}_{2} \mathrm{OCH}_{2}\right), 2.96\left(2 \mathrm{H}, \mathrm{t}, J=6 \mathrm{~Hz}, \mathrm{CH}_{2} \mathrm{~N}\right), 2.71$ $\left(2 \mathrm{H}\right.$, brs, $\left.\mathrm{CH}_{2} \mathrm{~N}\right), 2.52\left(2 \mathrm{H}\right.$, brs, $\left.\mathrm{CH}_{2} \mathrm{~N}\right) .{ }^{13} \mathrm{CNMR}(75 \mathrm{MHz}$, DMSO- $\left.d_{6}\right) \delta /$ ppm: $163.3,163.0,155.0,134.0,124.6,116.7$, 116.0, 98.9, 65.8, 65.5, 56.6, 53.6, 53.5. Anal. Calcd. $w / \%$ for $\mathrm{C}_{17} \mathrm{H}_{20} \mathrm{~N}_{2} \mathrm{O}_{4}$ (316.36): C, 64.54; $\mathrm{H}, 6.37 ; \mathrm{N}, 8.86$; Found: C, 64.49; H, 6.43; N, 8.81 .

Methyl (E)-2-cyano-3-(3-(2-morpholinoethoxy)phenyl)acrylate (3q): m.p. $180-183^{\circ} \mathrm{C}$; IR (KBr) $\left(v_{\max } / \mathrm{cm}^{-1}\right)$ : 2953, 2923, 2852, 2224, 1734, 1610, 1580, 1437, 1243, 1130, 954, 684, 515. ${ }^{1} \mathrm{HNMR}\left(300 \mathrm{MHz}, \mathrm{DMSO}-d_{6}\right) \delta / \mathrm{ppm}: 8.40(1 \mathrm{H}, \mathrm{s}$, $\left.\mathrm{CH}_{\text {vinylic }}\right), 7.69\left(1 \mathrm{H}, \mathrm{d}, J=9 \mathrm{~Hz}, \mathrm{CH}_{\mathrm{Ar}}\right), 7.67\left(1 \mathrm{H}, \mathrm{s}, \mathrm{CH}_{\mathrm{Ar}}\right), 7.53$ $\left(1 \mathrm{H}, \mathrm{t}, J=9 \mathrm{~Hz}, \mathrm{CH}_{\mathrm{Ar}}\right), 7.28\left(1 \mathrm{H}, \mathrm{d}, J=9 \mathrm{~Hz}, \mathrm{CH}_{\mathrm{Ar}}\right), 4.25(2 \mathrm{H}$, $\left.\mathrm{t}, J=6 \mathrm{~Hz}, \mathrm{OCH}_{2}\right), 3.88\left(3 \mathrm{H}, \mathrm{s}, \mathrm{OCH}_{3}\right), 3.67(4 \mathrm{H}, \mathrm{t}, J=3 \mathrm{~Hz}$, $\left.\mathrm{CH}_{2} \mathrm{OCH}_{2}\right), 2.94\left(2 \mathrm{H}, \mathrm{t}, J=6 \mathrm{~Hz}, \mathrm{CH}_{2} \mathrm{~N}\right), 2.70(4 \mathrm{H}, \mathrm{brs}$, $\mathrm{CH}_{2} \mathrm{NCH}_{2}$ ). ${ }^{13} \mathrm{CNMR}$ (75 MHz, DMSO-d $\left.d_{6}\right) \delta / \mathrm{ppm}: 162.7$, $158.9,155.5,133.0,130.9,123.7,120.4,116.9,116.0$, 103.0, 65.8, 65.2, 56.7, 53.8, 53.5. Anal. Calcd. $w / \%$ for $\mathrm{C}_{17} \mathrm{H}_{20} \mathrm{~N}_{2} \mathrm{O}_{4}$ (316.36): C, 64.54; $\mathrm{H}, 6.37 ; \mathrm{N}, 8.86$; Found: $\mathrm{C}$, $64.61 ; \mathrm{H}, 6.39 ; \mathrm{N}, 8.92$.

Methyl (E)-3-(4-(4-bromobenzyl)oxy)phenyl)-2-cyanoacrylate (3r): m.p. $129-132{ }^{\circ} \mathrm{C}$; IR $(\mathrm{KBr})\left(v_{\max } / \mathrm{cm}^{-1}\right): 3027,2948$, 2218, 1724, 1590, 1514, 1434, 1311, 1268, 1214, 1184, 1094, 1070, 804, 759, 515. ${ }^{1} \mathrm{HNMR}\left(300 \mathrm{MHz}, \mathrm{CDCl}_{3}\right) \delta /$ ppm: $8.21\left(1 \mathrm{H}, \mathrm{s}, \mathrm{CH}_{\text {vinylic }}\right), 8.03\left(2 \mathrm{H}, \mathrm{d}, J=9 \mathrm{~Hz}, \mathrm{CH}_{\mathrm{Ar}}\right), 7.57$ $\left(2 \mathrm{H}, \mathrm{d}, J=9 \mathrm{~Hz}, \mathrm{CH}_{\mathrm{Ar}}\right), 7.34\left(2 \mathrm{H}, \mathrm{d}, J=9 \mathrm{~Hz}, \mathrm{CH}_{\mathrm{Ar}}\right), 7.07(2 \mathrm{H}$, $\left.\mathrm{d}, J=9 \mathrm{~Hz}, \mathrm{CH}_{\mathrm{Ar}}\right), 5.13\left(2 \mathrm{H}, \mathrm{s}, \mathrm{OCH}_{2}\right), 3.95\left(3 \mathrm{H}, \mathrm{s}, \mathrm{OCH}_{3}\right)$. ${ }^{13} \mathrm{CNMR}\left(75 \mathrm{MHz}, \mathrm{CDCl}_{3}\right.$ ) $\delta / \mathrm{ppm}: 163.5,162.6,154.5$, 134.8, 133.7, 131.9, 129.1, 124.7, 122.3, 116.1, 115.5, 99.2, 69.5, 53.2. Anal. Calcd. $w / \%$ for $\mathrm{C}_{18} \mathrm{H}_{14} \mathrm{BrNO}_{3}$ (372.22): $\mathrm{C}$, 58.08; H, 3.79; N, 3.76; Found: C, 58.11; H, 3.85; N, 3.81.

Methyl (E)-3-(4-((4-chlorobenzyl)oxy)phenyl)-2-cyanoacrylate (3s): m.p. $142-145^{\circ} \mathrm{C}$; IR $(\mathrm{KBr})\left(v_{\max } / \mathrm{cm}^{-1}\right)$ : 3028, 2951, 2219, 1725, 1589, 1515, 1433, 1387, 1311, 1267, 1214, 1184, 1093, 1005, 831, 759, 563, 514. ${ }^{1} \mathrm{HNMR}(300 \mathrm{MHz}$,
$\left.\mathrm{CDCl}_{3}\right) \delta /$ ppm: $8.21\left(1 \mathrm{H}, \mathrm{s}, \mathrm{CH}_{\text {vinylic }}\right), 8.03(2 \mathrm{H}, \mathrm{d}, J=9 \mathrm{~Hz}$, $\left.\mathrm{CH}_{\mathrm{Ar}}\right), 7.41\left(4 \mathrm{H}, \mathrm{brs}, \mathrm{CH}_{\mathrm{Ar}}\right), 7.07\left(2 \mathrm{H}, \mathrm{d}, J=9 \mathrm{~Hz}, \mathrm{CH}_{\mathrm{Ar}}\right), 5.15$ $\left(2 \mathrm{H}, \mathrm{s}, \mathrm{OCH}_{2}\right), 3.95\left(3 \mathrm{H}, \mathrm{s}, \mathrm{OCH}_{3}\right) .{ }^{13} \mathrm{CNMR}\left(75 \mathrm{MHz} \mathrm{CDCl}_{3}\right) \delta$ / ppm: 163.5, 162.6, 154.5, 134.3, 134.2, 133.7, 128.9, 128.8, 124.7, 116.1, 115.5, 99.2, 69.5, 53.2. Anal. Calcd. $w / \%$ for $\mathrm{C} 18 \mathrm{H} 14 \mathrm{CINO} 3$ (327.76): C, 65.96; H, 4.31; N, 4.27; Found: C, 65.91; H, 4.25; N, 4.36 .

Methyl (E)-3-(3-((4-chlorobenzyl)oxy)phenyl)-2-cyanoacrylate (3t): m.p. 83-86 ${ }^{\circ} \mathrm{C}$; IR ( $\left.\mathrm{KBr}\right)\left(v_{\max } / \mathrm{cm}^{-1}\right)$ : 2955, 2918, 2223, 1730, 1607, 1576, 1492, 1445, 1267, 1247, 1180, 1095, 804, 679. ${ }^{1} \mathrm{HNMR}\left(300 \mathrm{MHZ}, \mathrm{CDCl}_{3}\right) \delta / \mathrm{ppm}: 8.25\left(1 \mathrm{H}, \mathrm{s}, \mathrm{CH}_{\text {vinylic }}\right)$, $7.71\left(1 \mathrm{H}, \mathrm{s}, \mathrm{CH}_{\mathrm{Ar}}\right), 7.53\left(1 \mathrm{H}, \mathrm{d}, J=9 \mathrm{~Hz}, \mathrm{CH}_{\mathrm{Ar}}\right), 7.44(1 \mathrm{H}, \mathrm{t}, J=$ $\left.9 \mathrm{~Hz}, \mathrm{CH}_{\mathrm{Ar}}\right), 7.41-7.38\left(4 \mathrm{H}, \mathrm{m}, \mathrm{CH}_{\mathrm{Ar}}\right), 7.20(1 \mathrm{H}, \mathrm{d}, J=9 \mathrm{~Hz}$, $\left.\mathrm{CH}_{\mathrm{Ar}}\right), 5.13\left(2 \mathrm{H}, \mathrm{s}, \mathrm{OCH}_{2}\right), 3.97\left(3 \mathrm{H}, \mathrm{s}, \mathrm{OCH}_{3}\right) .{ }^{13} \mathrm{CNMR}(75$ $\left.\mathrm{MHz}, \mathrm{CDCl}_{3}\right) \delta / \mathrm{ppm}: 162.9,158.8,155.1,134.7,134.0$, $132.6,130.4,128.9,128.8,124.9,121.1,115.5,115.2$, 102.8, 69.4, 53.4. Anal. Calcd. $w / \%$ for $\mathrm{C}_{18} \mathrm{H}_{14} \mathrm{ClNO}_{3}$ (327.76): C, 65.96; H, 4.31; N, 4.27; Found: C, 65.91; H, 4.25; $\mathrm{N}, 4.36$.

Ethyl (E)-2-cyano-3-(4-(4-phenylpiperidin-1-yl)phenyl)acrylate (3x): m.p. $115-116{ }^{\circ} \mathrm{C}$; IR $(\mathrm{KBr})\left(v_{\max } / \mathrm{cm}^{-1}\right)$ : 3021, 2992, 2933, 2208, 1727, 1609, 1563, 1510, 1274, 1226, 1172, 964, $812,751,705,517$; ${ }^{1} \mathrm{HNMR}$ (300 MHZ, $\mathrm{CDCl}_{3}$ ) $\delta$ / ppm: 8.10 (s, $\left.1 \mathrm{H}, \mathrm{CH}_{\text {Vinylic }}\right), 7.96\left(\mathrm{~d}, 2 \mathrm{H}, J=9 \mathrm{~Hz}, \mathrm{CH}_{\mathrm{Ar}}\right), 7.36-7.18(\mathrm{~m}$, $\left.5 \mathrm{H}, \mathrm{CH}_{\mathrm{Ph}}\right), 6.89\left(\mathrm{~d}, J=9 \mathrm{~Hz}, 2 \mathrm{H}, \mathrm{CH}_{\mathrm{Ar}}\right), 4.38(\mathrm{q}, J=6 \mathrm{~Hz}, 2 \mathrm{H}$, $\mathrm{OCH}_{2}$ ), 3.99 (d, $J=9 \mathrm{~Hz}, 2 \mathrm{H}, \mathrm{CH}_{\mathrm{Ar}}$ ), 2.93 (d.t, $2 \mathrm{H},{ }^{1} \mathrm{~J}=9 \mathrm{~Hz},{ }^{2} \mathrm{~J}$ $=3 \mathrm{~Hz}, 2 \mathrm{CH}), 2.61\left(\mathrm{~d}, J=6 \mathrm{~Hz}, 2 \mathrm{H}, \mathrm{CH}_{2}\right) 1.92-1.86(\mathrm{~m}, 1 \mathrm{H}$, $\mathrm{CH}), 1.81(\mathrm{~d}, J=12 \mathrm{~Hz}, 2 \mathrm{H}, 2 \mathrm{CH}), 1.41\left(\mathrm{t}, J=6 \mathrm{~Hz}, 3 \mathrm{H}, \mathrm{CH}_{3}\right)$, $1.35(\mathrm{~d}, J=3 \mathrm{~Hz}, 2 \mathrm{H}, 2 \mathrm{CH}) .{ }^{13} \mathrm{CNMR}\left(75 \mathrm{MHz}, \mathrm{CDCl}_{3}\right) \delta / \mathrm{ppm}$ : $164.2,154.3,153.9,139.9,134.1,129.1,128.4,126.1$, 120.3, 117.4, 113.4, 94.9, 81.9, 47.5, 42.9, 38.1. 31.5, 14.3 . Anal. Calcd. $w / \%$ for $\mathrm{C}_{24} \mathrm{H}_{26} \mathrm{~N}_{2} \mathrm{O}_{2}$ : C, 76.98; H, 7.00; N, 7.48; Found: C, 77.01; H, 7.13; N, 7.51.

Ethyl (E)-2-cyano-3-(4-(2-morpholinoethoxy)phenyl)acrylate (3y): m.p. $217-218{ }^{\circ} \mathrm{C}$; IR $(\mathrm{KBr})\left(v_{\max } / \mathrm{cm}^{-1}\right)$ : 3033, 2996, 2938, 2217, 1718, 1588, 1514, 1263, 1185, 940, 834, 517; ${ }^{1} \mathrm{HNMR}\left(300 \mathrm{MHZ}, \mathrm{CDCl}_{3}\right) \delta / \mathrm{ppm}: 8.32\left(\mathrm{~s}, 1 \mathrm{H}, \mathrm{CH}_{\text {vinylic }}\right), 8.09$ (d, $\left.J=9 \mathrm{~Hz}, 2 \mathrm{H}, \mathrm{CH}_{\mathrm{Ar}}\right), 7.18\left(\mathrm{~d}, J=9 \mathrm{~Hz}, 2 \mathrm{H}, \mathrm{CH}_{\mathrm{Ar}}\right), 4.31$ (q, J $=6 \mathrm{~Hz}, 2 \mathrm{H}, \mathrm{OCH}_{2}$ ), 4.30 (brs, $2 \mathrm{H}, \mathrm{CH}_{2}$ ), 3.66 (brs, $4 \mathrm{H}, 2 \mathrm{CH}_{2}$ ), $2.92\left(\mathrm{brs}, 2 \mathrm{H}, \mathrm{CH}_{2}\right), 2.68\left(\mathrm{brs}, 4 \mathrm{H}, 2 \mathrm{CH}_{2}\right), 1.32(3 \mathrm{H}, \mathrm{t}, J=6 \mathrm{~Hz}$, $\left.\mathrm{CH}_{3}\right) .{ }^{13} \mathrm{CNMR}\left(75 \mathrm{MHz}, \mathrm{CDCl}_{3}\right.$ ) $\delta / \mathrm{ppm}: 163.0,162.8,154.9$, 133.9, 124.6, 116.7, 115.9, 99.2, 65.9, 65.6, 62.6, 56.7, 53.5, 14.5. Anal. Calcd. $w / \%$ for $\mathrm{C}_{18} \mathrm{H}_{22} \mathrm{~N}_{2} \mathrm{O}_{4}: \mathrm{C}, 65.44 ; \mathrm{H}, 6.71 ; \mathrm{N}$, 8.48. Found: C, 65.49; H, 6.68; N, 8.50.

Ethyl (E)-3-(4-((4-bromobenzyl)oxy)phenyl)-2-cyanoacrylate (3z): m.p. $140-142{ }^{\circ} \mathrm{C}$; IR $(\mathrm{KBr})\left(v_{\max } / \mathrm{cm}^{-1}\right)$ : 3027, 2948, 2218, 1724, 1590, 1514, 1434, 1381, 1311, 1268, 1214, 1183, 1093, 1069, 1002, 832, 804, 759, 554, 515. ${ }^{1} \mathrm{HNMR}$ ( $300 \mathrm{MHz}, \mathrm{CDCl}_{3}$ ) $\delta / \mathrm{ppm}: 8.20\left(\mathrm{~s}, 1 \mathrm{H}, \mathrm{CH}_{\text {Vinylic }}\right), 8.03$ (d, $J=$ $\left.9 \mathrm{~Hz}, 2 \mathrm{H}, \mathrm{CH}_{\mathrm{Ar}}\right), 7.57\left(\mathrm{~d}, J=9 \mathrm{~Hz}, 2 \mathrm{H}, \mathrm{CH}_{\mathrm{Ar}}\right), 7.34(\mathrm{~d}, J=9 \mathrm{~Hz}$, $\left.2 \mathrm{H}, \mathrm{CH}_{\mathrm{Ar}}\right), 7.08\left(\mathrm{~d}, J=9 \mathrm{~Hz}, 2 \mathrm{H}, \mathrm{CH}_{\mathrm{Ar}}\right), 5.13\left(\mathrm{~s}, 2 \mathrm{H}, \mathrm{OCH}_{2}\right)$, 
$4.40\left(\mathrm{q}, J=6 \mathrm{~Hz}, 2 \mathrm{H}, \mathrm{OCH}_{2}\right), 1.42\left(\mathrm{t}, J=6 \mathrm{~Hz}, 3 \mathrm{H}, \mathrm{CH}_{3}\right)$. ${ }^{13} \mathrm{CNMR}\left(75 \mathrm{MHz}, \mathrm{CDCl}_{3}\right.$ ) $\delta / \mathrm{ppm}: 163.0,162.5,154.2$, 134.8, 133.6, 131.9, 129.1, 124.8, 122.3, 116.1, 115.5, 99.8, 69.5, 62.5, 14.2. Anal. Calcd. $w / \%$ for $\mathrm{C}_{19} \mathrm{H}_{16} \mathrm{BrNO}_{3}$ : C, 59.08; H, 4.18; N, 3.63; Found: C, 59.12; H, 4.11; N, 3.67.

\section{RESULTS AND DISCUSSION}

Silica sodium carbonate nanoparticles were synthesized by a slight modification of the solvothermal reported method. ${ }^{[43]}$ The FT-IR spectrum of SSC NPs are exactly same as what reported in the literature ${ }^{[43]}$ and strongly confirm the catalyst's structure. It should be mentioned that all important peaks centered at $3426,1626,1453,1082,880,797$ and $468 \mathrm{~cm}^{-1}$ are obviously seen in the FT-IR spectrum of SSC NPs (supplementary materials).

The morphology of SSC NPs was studied by scanning electron microscopy (SEM) (Figure 1). SEM image of SSC NPs shows the silica sodium carbonate nanoparticles have a diameter of about $30 \mathrm{~nm}$ without any amorphous or other kinds of crystallized phase particles. In addition, the transmission electron microscopy (TEM) image of silica sodium carbonate nanoparticles was studied for more investigation of SSC NPs morphology (Figure 2). TEM image of SSC NPS clearly demonstrated the SSC NPs have a homogeneous diameter size about $30 \mathrm{~nm}$.

In another investigation, to quantify the exact amount of the sodium carbonate groups attached to the silica, the catalyst was titrated with $\mathrm{HCl}(0.01 \mathrm{~N})$. Considering to the molecular weight of $\mathrm{CO}_{3} \mathrm{Na}(83 \mathrm{~g})$, the results demonstrated that $0.006 \mathrm{~g}$ of SSC NPs was equivalent to 0.01 $\mathrm{mmol} \mathrm{CO}{ }_{3} \mathrm{Na}$. In other words, $0.006 \mathrm{~g}$ of catalyst is containing $1 \mathrm{~mol} \%$ of $\mathrm{CO}_{3} \mathrm{Na}$ group.

Also it is interesting to note that the reproducibility of the SSC nanoparticle synthesis when considering the percentage of the attached sodium carbonate groups was very good so that titration results with $\mathrm{HCl}(0.01 \mathrm{~N})$ for reproduced catalysts were very close to previous one. From titration results on reproduced catalysts, it could generally be concluded that $0.006 \mathrm{~g}$ of catalyst was equivalent to 0.01 $\mathrm{mmol} \mathrm{CO}_{3} \mathrm{Na}$.

Before starting to develop reaction scope by the use of numerous arylaldehydes and malononitrile derivatives, it was tried to find best condition to obtain products with highest yield in shortest reaction time on model reaction (compound 3a) and the results were summarized in Table 1.

First of all, the reaction was loaded in catalyst-free conditions. It was observed that no remarkable product was obtained in absence of catalyst. Among examined solvents, target product was obtained with more yields in the presence of acetonitrile. In addition, the application of sonication significantly leads to increase of reaction yield. As

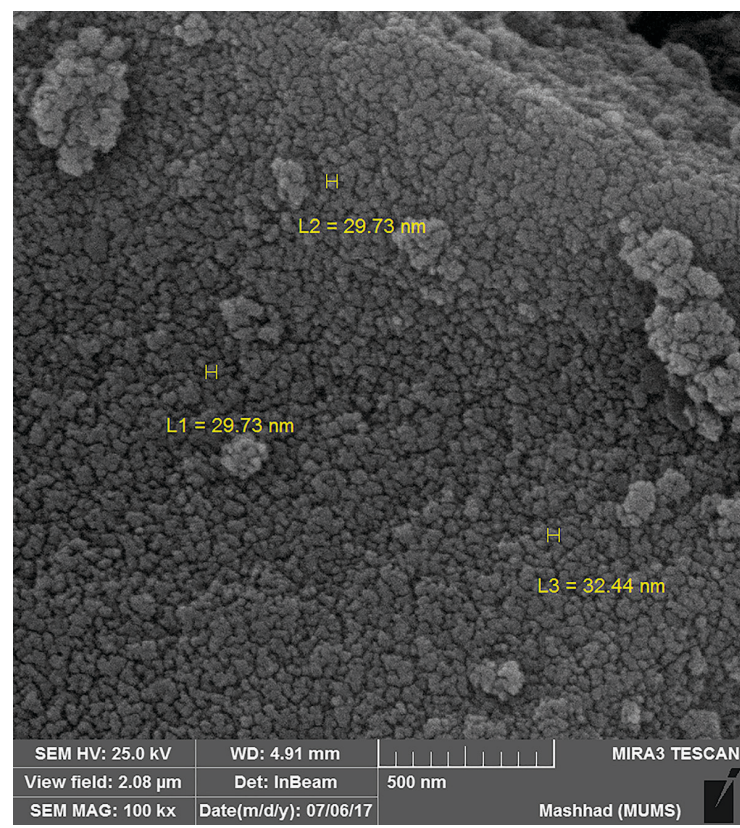

Figure 1. SEM image of SSC NPS.

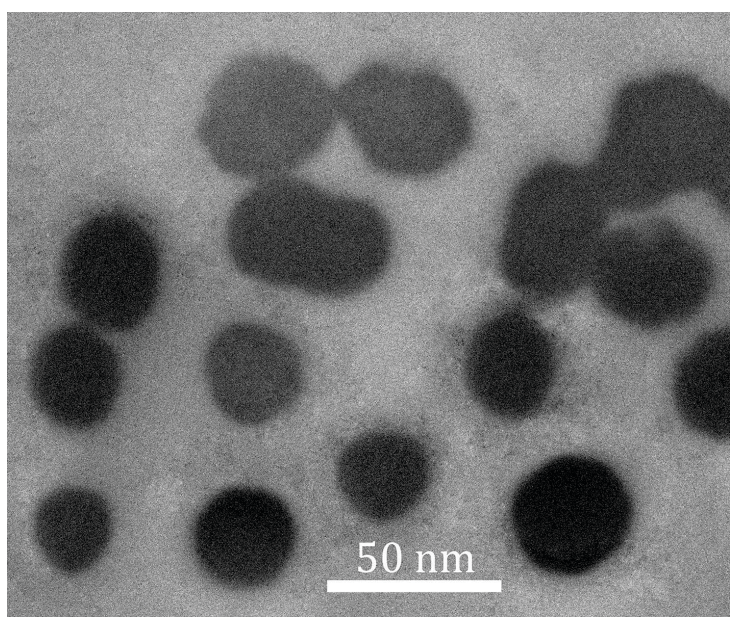

Figure 2. TEM image of SSC NPS.

well as, the use of SSC NPs instead of $\mathrm{SiO}_{2}$ or SSC bulk resulted to remarkably increase of reaction yield. As can be seen from Table 1, among investigated of different conditions, using SSC NPs ( 2 mol\%) under sonication irradiation at $70{ }^{\circ} \mathrm{C}$ was selected as the best condition to obtain products with highest yields.

After finding the optimum conditions for reaction performance, the generality of the reaction was examined by the use of a wide range of arylaldehydes and malononitrile or methyl/ethyl cianoacetate in the presence of optimum amounts of SSC NPs as green, highly efficient and recyclable nano-catalyst, and the results were summarized in Table 2. ${ }^{[47-50]}$ 
Table 1. Investigation of different conditions on model reaction ${ }^{(a)}$

\begin{tabular}{|c|c|c|c|}
\hline Entry & Catalyst & Conditions & Yield $^{(b)}$ \\
\hline 1 & None & $\mathrm{CH}_{2} \mathrm{Cl}_{2}, 40^{\circ} \mathrm{C}$ & 21 \\
\hline 2 & None & $\mathrm{H}_{2} \mathrm{O}, 70^{\circ} \mathrm{C}$ & 33 \\
\hline 3 & None & $\mathrm{THF}, 66^{\circ} \mathrm{C}$ & 40 \\
\hline 4 & None & $\mathrm{EtOH}, 50^{\circ} \mathrm{C}$ & 38 \\
\hline 5 & None & $\mathrm{EtOH}, 60^{\circ} \mathrm{C}$ & 42 \\
\hline 6 & None & $\mathrm{EtOH}, 70{ }^{\circ} \mathrm{C}$ & 45 \\
\hline 7 & None & $\mathrm{CH}_{3} \mathrm{CN}, 70{ }^{\circ} \mathrm{C}$ & 49 \\
\hline 8 & $\mathrm{SiO}_{2}(0.018 \mathrm{~g})$ & $\mathrm{CH}_{3} \mathrm{CN}, 70^{\circ} \mathrm{C}$ & 60 \\
\hline 9 & SSC bulk (1 mol\%) & $\mathrm{CH}_{3} \mathrm{CN}, 70{ }^{\circ} \mathrm{C}$ & 69 \\
\hline 10 & SSC bulk (1 mol\%) & $\mathrm{CH}_{3} \mathrm{CN}$, Sonication, $70^{\circ} \mathrm{C}$ & 75 \\
\hline 11 & SSC bulk ( 2 mol\%) & $\mathrm{CH}_{3} \mathrm{CN}$, Sonication, $70^{\circ} \mathrm{C}$ & 86 \\
\hline 12 & SSC NPs (1 mol\%) & $\mathrm{CH}_{3} \mathrm{CN}$, Sonication, $70^{\circ} \mathrm{C}$ & 88 \\
\hline 13 & SSC NPs (2 mol\%) & $\mathrm{CH}_{3} \mathrm{CN}$, Sonication, $70^{\circ} \mathrm{C}$ & 98 \\
\hline 14 & SSC NPs (3 mol\%) & $\mathrm{CH}_{3} \mathrm{CN}$, Sonication, $70^{\circ} \mathrm{C}$ & 98 \\
\hline 15 & SSC NPs (4 mol\%) & $\mathrm{CH}_{3} \mathrm{CN}$, Sonication, $50^{\circ} \mathrm{C}$ & 97 \\
\hline
\end{tabular}

(a) Reaction time: 25 min

(b) Isolated yield

As can be seen from Table 2, all products bearing both electron donating/withdrawing groups on arylaldehyde rings under optimum conditions resulted to obtain compound $\mathbf{3}$ with excellent yields. Also it may be noticed to the reports on Knoevenagel condensation reactions catalyzed by some standard commercially available mesoporous silica such as functionalized MCM-48 or SBA-15. [51] However MCM-48 or SBA-15 themselves are not as efficient as SSC NPs in Knoevenagel condensation reaction but when they would functionalize by amino groups or ionic liquids they can almost act as good as SSC NPs in above condensation reaction. However commercial samples of MCM-48 or SBA-15 are rather expensive than SSC NPS

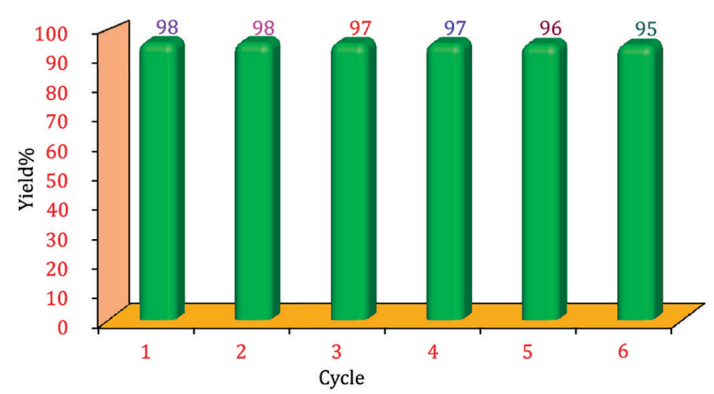

Figure 3. Reusability results of SSC NPs on model reaction over six cycles; Reaction conditions: 4-nitrobenzaldehyde (1 mmol, $0.151 \mathrm{~g})$, malononitrile (1.5 mmol $0.099 \mathrm{~g})$, SSC NPs (2 mol\%), $\mathrm{CH}_{3} \mathrm{CN}(10 \mathrm{~mL})$, Sonication, $70{ }^{\circ} \mathrm{C}$. which it is indeed the significantly lower cost catalyst than MCM-48 or SBA-15.

\section{Reusability of the Catalyst}

Because of the capability of catalyst to reuse makes it industrially and economically considerable and can be considered as one of the noteworthy aspects of this methodology, the capability of SSC NPs to reuse was evaluated on model reaction and the results were given in Figure 3 . This investigation reveals that SSC NPs can efficiently catalyze the reaction up to six times without significant loss of activity.

\section{CONCLUSION}

In summary, an efficient nano-catalytic approach to the synthesis of newly prepared arylidene malononitriles or methy/ethyl cianoacetates was described by the use of SSC NPs as a highly efficient nano-catalyst. By this achievement some novel products were obtained which can be candidate as medicinally important compounds or even important drugs in the future. Therefore, this methodology can attract the interest of chemists, biologists and pharmacologists in the future. In addition, a new application of SSC NPs as a green, highly efficient, reusable, and easily handled nano-catalyst to the synthesis of novel organic compounds was successfully examined. All products were isolated in excellent yields, and the catalyst efficiently recovered and successfully reused in further cycles. 
Table 2. Highly efficient and practical synthesis of compounds 3a-z via Knoevenagel condensation reaction between arylaldehydes and malononitrile or ethyl/methyl cianoacetate(a)

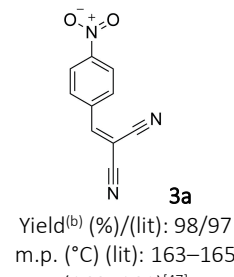

$(160-161)^{[47]}$

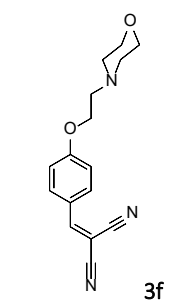

Yield ${ }^{(b)}(\%): 92$ m.p. $\left({ }^{\circ} \mathrm{C}\right): 228-229^{(c)}$

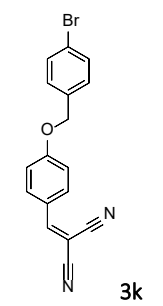

Yield $^{(b)}(\%): 95$ m.p. $\left({ }^{\circ} \mathrm{C}\right): 187-190^{(c)}$

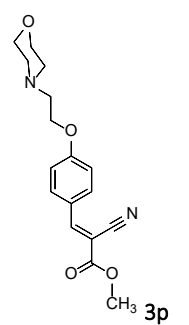

Yield(b) $(\%): 91$ m.p. $\left({ }^{\circ} \mathrm{C}\right): 203-207^{(c)}$

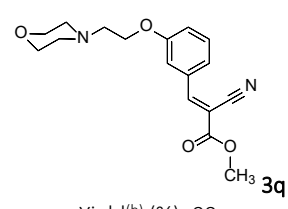

Yield $^{(b)}(\%): 90$ m.p. $\left({ }^{\circ} \mathrm{C}\right): 180-183^{(\mathrm{c})}$

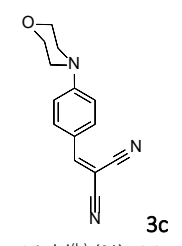

Yield(b) $(\%): 94$ m.p. $\left({ }^{\circ} \mathrm{C}\right): 198-200^{(c)}$

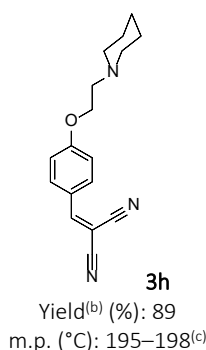

m.p. $\left({ }^{\circ} \mathrm{C}\right): 195-198^{(c)}$

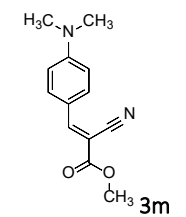

Yield $^{(b)}(\%) /($ lit): 95/91 m.p. $\left({ }^{\circ} \mathrm{C}\right)$ (lit): $148-149$ $(145)^{[49]}$

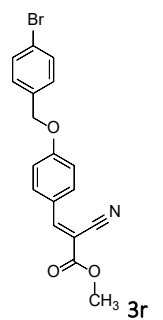

Yield(b) (\%): 95

m.p. $\left({ }^{\circ} \mathrm{C}\right): 129-132^{(\mathrm{c})}$

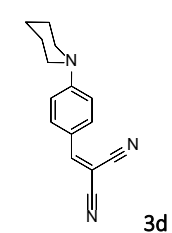

Yield(b) $^{(\%): ~} 93$ m.p. $\left({ }^{\circ} \mathrm{C}\right): 134-135^{\text {(c) }}$

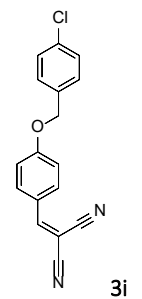

Yield ${ }^{(b)}(\%): 97$ m.p. $\left({ }^{\circ} \mathrm{C}\right): 174-176^{(c)}$

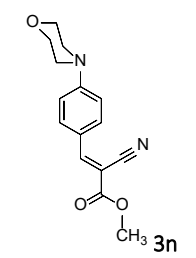

Yield $^{(\text {b) }}(\%) /($ lit): 93/84 m.p. $\left({ }^{\circ} \mathrm{C}\right)$ (lit): $146-149$ (not reported) ${ }^{[50]}$

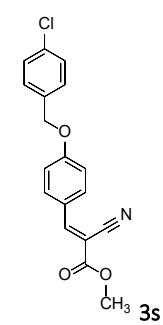

Yield $^{(\text {b) }}(\%): 94$ m.p. $\left({ }^{\circ} \mathrm{C}\right): 142-145^{(\mathrm{c})}$
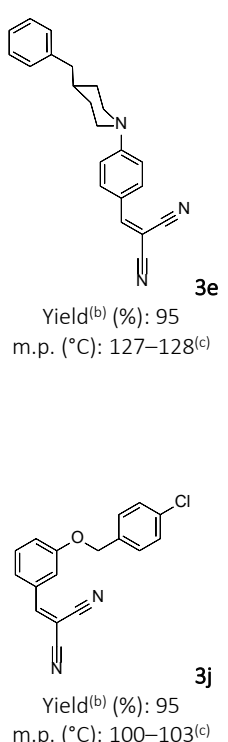

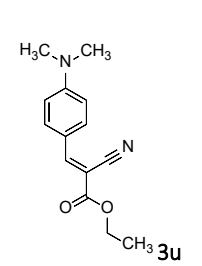

Yield $^{(b)}(\%) /($ lit): 98/96 m.p. $\left({ }^{\circ} \mathrm{C}\right)$ (lit): $127-129$ $(123-125)^{[48]}$

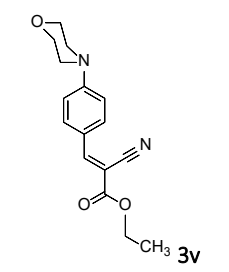

Yield $^{(b)}(\%) /($ lit): $94 / 86$

m.p. $\left({ }^{\circ} \mathrm{C}\right.$ ) (lit): $155-157$ (not reported) ${ }^{[50]}$

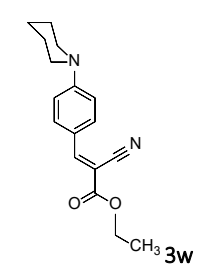

Yield $^{(\text {b) }}(\%) /($ lit): 93/90

m.p. $\left({ }^{\circ} \mathrm{C}\right)$ (lit): $90-92$ (not reported) ${ }^{[50]}$

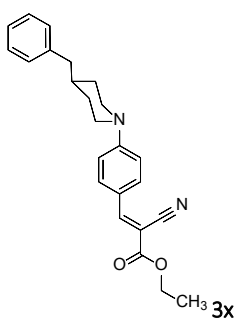

Yield(b) (\%): 95 m.p. $\left({ }^{\circ} \mathrm{C}\right): 115-116^{(c)}$

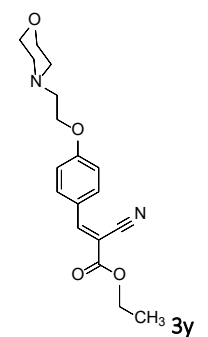

Yield(b) $(\%): 93$ m.p. $\left({ }^{\circ} \mathrm{C}\right): 217-218^{(c)}$

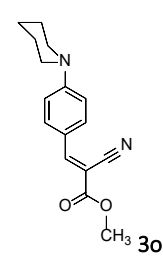

Yield $^{(b)}(\%): 90$ m.p. $\left({ }^{\circ} \mathrm{C}\right): 96-99^{(c)}$

\footnotetext{
(a) Reaction conditions: SSC NPs (2 mol\%), $\mathrm{CH}_{3} \mathrm{CN}(10 \mathrm{~mL})$, Sonication, $70^{\circ} \mathrm{C}$

(b) Isolated yields.

(c) Novel product.
} 
Acknowledgment. Financial support from Pharmaceutics Research Center, Institute of Neuropharmacology, Kerman University of Medical Sciences is greatly acknowledged.

Supplementary Information. Supporting information to the paper is attached to the electronic version of the article at: http://doi.org/10.5562/cca3261.

\section{REFERENCES}

[1] T. J. Schmidt, M. Ak, U. Mrowietz, Bioorg. Med. Chem. 2007, 15, 333.

[2] M. Tang, D. Shen, Y. Hu, S. Gao, S. Yu, J. Nat. Prod. 2004, 67, 1969.

[3] W. H. Dong, J. C. Chen, Y. L. He, J. J. Xu, Y. A. Mei, Am. J. Physiol. Cell Physiol. 2013, 305, 547.

[4] K. Eskandari, M. Rafieian-Kopaei, Chem. Heterocycl. Compd. 2016, 52, 158.

[5] J. G. Devassy, I. D. Nwachukwu, P. J. Jones, Nut. Rev. 2015, 73, 155.

[6] (a) E. Knoevenagel, Ber. Dtsch. Chem. Ges. 1894, 27, 2345; (b) E. Knoevenagel, Ber. Dtsch. Chem. Ges., 1898, 31, 2585.

[7] A. Taher, D. J. Lee, B. K. Lee, I. M. Lee, Synlett, 2016, 27, A.

[8] R. K. G. Panicker, S. Krishnapillai, Tetrahedron Lett. 2014, 55, 2352.

[9] (a) F. L. Yu, X. Y. He, C. Gu, E. Ohkoshi, L. T. Wang, S. B. Wang, C. Y. Lai, L. Yu, S. L. Morris-Natschke, K. H. Lee, S. Liu, L. Xie, Bioorg. Med. Chem. 2014, 22, 325; (b) K. Eskandari, B. Karami, Monatsh. Chem. 2016, 147, 2119; (c) K. Eskandari, B. Karami, S. Khodabakhshi, J. Chem. Res. 2014, 38, 600.

[10] (a) M. A. Camerino, M. Liu, S. Moriya, T. Kitahashi, A. Mahgoub, S. J. Mountford, D. K. Chalmers, T. Soga, I.

S. Parhar, P. E. Thompson, J. Pept. Sci. 2016, 22, 406; (b) B. Karami, K. Eskandari, M. Azizi, Lett. Org. Chem. 2013, 10, 722; (c) I. Karlsson, E. Persson, J. Martensson, A. Börje, Photochem. Photobiol. 2012, 88, 904.

[11] T. W. G. Solomons, C. Fryhle, Organic Chemistry; Wiley: New York, (2009).

[12] L. Poorali, B. Karami, K. Eskandari, M. Azizi, J. Chem. Sci. 2013, 125, 591.

[13] K. Eskandari, B. Karami, Comb. Chem. High Throughput Screening, 2016, 19, 728.

[14] A. Zhu, R. Liu, L. Li, L. Li, L. Wang, J. Wang, Catal. Today, 2013, 200, 17

[15] Y. F. Lai, H. Zheng, S. J. Chai, P. F. Zhang, X. Z. Chen, Green Chem. 2010, 12, 1917.

[16] M. M. H. Bhuiyan, M. I. Hossain, M. Ashraful, M. M. Mahmud, J. Chem. 2012, 2, 30.

[17] (a) J. McNulty, J. A. Steere, S. Wolf, Tetrahedron Lett.
1998, 39, 8013; (b) G. Palmisano, F. Tibiletti, A. Penoni, F. Colombo, S. Tollari, D. Garella, S. Tagliapietra, G. Cravotto, Ultrason. Sonochem. 2011, 18,652

[18] G. Guo, E. A. Arvanitis, R. S. Pottorf, M. P. Player, J. Comb. Chem. 2003, 5, 408 .

[19] M. A. Pasha, K. Manjula, J. Saudi Chem. Soc. 2011, 15, 283.

[20] A. K. Mitra, N. Karchandhuri, A. De, J. Indian Chem. Soc. 2005, 82, 177.

[21] Y. Ding, X. Ni, M. Gu, S. Li, H. Huang, Y. Hu, Catal. Commun. 2015, 64, 101.

[22] G. Li, J. Xiao, W. Zhang, Green Chem. 2011, 13, 1828.

[23] K. Liu, Y. Xu, Z. Yao, H. N. Miras, Y. F. Song, ChemCatChem. 2016, 8, 929.

[24] C. Wiles, P. Watts, S. J. Haswell, Chem. Commun., 2007, 2007, 966.

[25] W. B. Yi, Y. Q. Yin, C. Cai, Org. Prep. Proced. Int. 2007, 39, 71.

[26] A. Asadipour, Y. Pourshojaei, K. Eskandari, A. Foroumadi, RSC Adv. 2017, 7, 44680.

[27] N. Rodrigues, K. Bennis, D. Vivier, V. Pereira, F. C. Chatelain, E. Chapuy, H. Deokar, J. Busserolles, F. Lesage, A. Eschalier, S. Ducki, Eur. J. Med. Chem. 2014, 75, 391.

[28] D. Vivier, I. B. Soussia, N. Rodrigues, S. Lolignier, M. Devilliers, F. C. Chatelain, L. Prival, E. Chapuy, G. Bourdier, K. Bennis, F. Lesage, A. Eschalier, J. Busserolles, S. Ducki, J. Med. Chem. 2017, 60, 1076.

[29] C. Lerche, I. Bruhova, H. Lerche, K. Steinmeyer, A. D. Wei, N. Strutz-Seebohm, F. Lang, A. E. Busch, B. S. Zhorov, G. Seebohm, J. Med. Chem. 1991, 34, 1503.

[30] F. Horiuchi, K. Fujimoto, T. Ozaki, Y. Nishizawa, Agr. Bio. Chem. 1971, 35, 2003.

[31] P. Jimonet, Y. Ribeill, G. A. Bohme, A. Boireau, M. Cheve, D. Damour, A. Doble, A. Genevois-Borella, F. Herman, A. Imperato, S. Le-Guern, F. Manfre, J. Pratt, J. C. R. Randle, J. M. Stutzmann, S. Mignani, J. Med. Chem. 2000, 43, 2371.

[32] I. Karlsson, E. Persson, J. Martensson, A. Börje, Photochem. Photobiol. 2012, 88, 904.

[33] C. S. Bussche-Hunnefeld, R. Klintz, G. Hamprecht, E. Heistracher, P. Schafer, K. Ditrich, K. O. Westphalen, M. Gerber, H. Walter, US Patent 5744426 A, US5744426 A, Apr 28, (1998).

[34] H. Cho, M. Tamaoka, S. Murota, I. Morita, US Patent 5232941 A, US 07/750,396, Aug 3, (1993).

[35] J. Sutharsan, M. Dakanali, C. C. Capule, M. A. Haidekker, J. Yang, E. A. Theodorakis, Chem. Med. Chem. 2010, 5, 56.

[36] S. Hesse, G. Kirsch, Tetrahedron Lett. 2002, 43, 1213.

[37] D. Kong, Y. Liu, J. Zhang, H. Li, X. Wang, G. Liu, B. Li, Z. Xud, New J. Chem. 2014, 38, 3078. 
[38] B. Dutta, R. Schwarz, S. Omar, S. Natour, R. AbuReziq, Eur. J. Org. Chem. 2015, 2015, 1961.

[39] B. Karami, K. Eskandari, Z. Zare, S. Gholipour, Chem. Heterocycl. Compd. 2014, 49, 1715.

[40] A. D. Murkute, J. E. Jackson, D. J. Miller, J. Catal. 2011, 278, 189.

[41] B. Karami, R. Ferdosian, K. Eskandari, J. Chem. Res. 2014, 38, 41.

[42] J. Feng, Y. He, Y. Liu, Y. Du, D. Li, Chem. Soc. Rev. 2015, 44, 5291.

[43] K. Eskandari, B. Karami, S. Khodabakhshi, Catal. Commun. 2014, 54, 124.

[44] (a) S. Rahmani-Nezhad, L. Khosravani, M. Saeedi, K. Divsalar, L. Firoozpour, Y. Pourshojaei, Y. Sarrafi, H. Nadri, A. Moradi, M. Mahdavi, A. Shafiee, A. Foroumadi, Synth. Commun. 2015, 45, 751; (b) A. Z. Halimehjani, Y. Pourshojaei, M. R. Saidi, Tetrahedron Lett. 2009, 50, 32; (c) M. R. Saidi, Y. Pourshojaei, F. Aryanasab, Synth. Commun. 2009, 39, 1109; (d) A. Asadipour, Z. Shams, K. Eskandari, M. H. Moshafi, E. Faghih-Mirzaei, Y. Pourshojaei, Res. Chem. Int. 2018, 44, 1295.

[45] (a) F. Mehrabi, Y. Pourshojaei, A. Moradi, M. Sharifzadeh, L. Khosravani, R. Sabourian, S. Rahmani-Nezhad, M. Mohammadi-Khanaposhtani, M. Mahdavi, A. Asadipour, H. R. Rahimi, S. Moghimi,
A. Foroumadi, Future Med. Chem. 2017, 9, 659; (b) Y. Pourshojaei, A. Gouranourimi, S. Hekmat, A. Asadipour, S. Rahmani-Nezhad, A. Moradi, H. Nadri, F. H. Moghadam, S. Emami, A. Foroumadi, A. Shafiee, Eur. J. Med. Chem. 2015, 97, 181; (c) S. Rahmani-Nezhad, M. Safavi, M. Pordeli, S. K. Ardestani, L. Khosravani, Y. Pourshojaei, M. Mahdavi, S. Emami, A. Foroumadi, A. Shafiee, Eur. J. Med. Chem. 2014, 86, 562.

[46] (a) H. M. H. G. Albers, L. J. D. Hendrickx, R. J. P. VanTol, J. Hausmann, A. Perrakis, H. Ovaa, J. Med. Chem. 2011, 54, 4619; (b) R. Carrasco-Gomez, S. KeppnerWitter, M. Hieke, L. Lange, G. Schneider, M. Schubert-Zsilavecz, E. Proschak, B. Spankuch, Bioorg. Med. Chem. Lett. 2014, 24, 5063.

[47] X. Hao, P. Liyang, F. Xiaomin, L. Baoying, Z. Wenkai, L. Minghua, X. Yuanqing, D. Tao, C. Haibo, Tetrahedron Lett. 2017, 58, 2360.

[48] H. Kiyani, F. Ghorbani, Res. Chem. Int. 2015, 41, 7847.

[49] V. K. Gupta, R. A. Singh, RSC Adv. 2015, 5, 38591.

[50] H. Xu, X. Yu, L. Sun, J. Liu, W. Fan, Y. Shen, W. Wang, Tetrahedron Lett. 2008, 49, 4687.

[51] (a) S. G. Wang, Catal. Commun. 2003, 4, 469; (b) Y. Liu, J. Peng, S. Zhai, J. Li, J. Mao, M. Li, H. Qiu, G. Lai, Eur. J. Inorg. Chem. 2006, 2006, 2947. 